\title{
Changing the waveform of circadian rhythms: considerations for shift-work
}

\section{Elizabeth M. Harrison* and Michael R. Gorman}

Department of Psychology, Center for Chronobiology, University of California San Diego, La Jolla, CA, USA

\section{Edited by:}

Oscar Prospéro-García, Universidad

Nacional Autónoma de México,

Mexico

\section{Reviewed by:}

Joshi John, Center for Sleep

Research, University of California Los

Angeles, USA

Raul Aguilar-Roblero, Universidad

Nacional Autónoma de México,

Mexico

\section{${ }^{*}$ Correspondence:}

Elizabeth M. Harrison, Department of Psychology, University of California

San Diego, La Jolla, CA 92093-0109,

USA.

e-mail:emharrison@ucsd.edu
Circadian disruption in shift-work is common and has deleterious effects on health and performance. Current efforts to mitigate these harms reasonably focus on the phase of the circadian pacemaker, which unfortunately in humans, shifts slowly and often incompletely. Temporal reorganization of rhythmic waveform (i.e., the shape of its $24 \mathrm{~h}$ oscillation), rather than phase, however, may better match performance demands of shift-workers and can be quickly and feasibly implemented in animals. In fact, a bifurcated pacemaker waveform may permit stable entrainment of a bimodal sleep/wake rhythm promoting alertness in both night and daylight hours. Although bifurcation has yet to be formally assessed in humans, evidence of conserved properties of circadian organization and plasticity predict its occurrence: humans respond to conventional manipulations of waveform (e.g., photoperiodism); behaviorally, the sleep/wake rhythm is adaptable; and finally, the human circadian system likely derives from the same multiple cellular oscillators that permit waveform flexibility in the rodent pacemaker. In short, investigation into untried manipulations of waveform in humans to facilitate adjustment to challenging schedules is justified.

Keywords: waveform, shift-work, split schedules, dysrhythmia, night shift

Across mammalian species, daily rhythms in myriad biological processes are orchestrated through a master clock embodied in the suprachiasmatic nuclei (SCN) of the anterior hypothalamus (Dunlap et al., 2004). This neural pacemaker coordinates a daily alternation in physiological and behavioral states to correspond with the changing day/night environment, though it persists in the absence of external timing cues. The subjective night of mammals is characterized by lower SCN metabolic and electrical activity, sensitivity to phase-resetting actions of light, and high levels of melatonin secretion. The opposite characterize the subjective day. While these fundamental properties of the circadian clock are shared between nocturnal and diurnal animals, the alignment of other rhythmic outputs (e.g., sleep/wake, hormone secretion, and body temperature) to subjective day or night varies with the ecological niche of the organism: nocturnal animals (e.g., most rodents) have high levels of activity during the night while diurnal animals (e.g., humans) are programmed for increased activity during the subjective day, with sleep preferred during subjective night (Figure 1A). Among chronobiologists, this species-typical orchestration of bodily rhythms is considered essential for optimal health (Golombek and Rosenstein, 2010).

The paramount problem faced by human shift-workers is that work, typically requiring sustained attention and precluding sleep, is sometimes demanded of them during their subjective night. To a significant extent, shift-workers can consciously override the influences of the circadian pacemaker to remain awake at night and sleep during the day, albeit at reduced levels of alertness and quality, respectively (Akerstedt and Wright, 2009). Critically, this volitional adjustment of behavior does not typically produce a corresponding shift in the rhythm of the circadian pacemaker (Folkard, 2008). As a result, the worker is considered dysrhythmic - any given behavior or physiological measure may remain rhythmic, but the constellation of rhythms is misaligned with respect to the environment and/or one another (Figure 1B).

Such dysrhythmia in humans is a common problem in our $24 \mathrm{~h}$ society. An estimated $17-60 \%$ of Americans work outside the regular daytime shift (Rosekind, 2005; McMenamin, 2007), resulting in annual costs exceeding $\$ 200$ billion (Kerin and Aguirre, 2005). This estimate includes two cost categories: first, due to the mismatch between work schedule and alertness, night work carries an increased incidence of injuries, accidents, and errors compared to day work (Mitler et al., 1988; Folkard and Tucker, 2003); second, due to the physiological consequences of dysrhythmia, including mistimed sleep and/or light exposure, shift-work results in serious deficits in health (Costa, 1996) including increased cancer risk (Hansen, 2001; Costa et al., 2010). Persistent circadian perturbations can even increase mortality in rodents (Davidson et al., 2006). Mitigation of the harms of this mismatch between environment and physiology therefore has the potential to increase the safety, productivity, and health of the shift-worker.

\section{CURRENT TREATMENTS TARGET CIRCADIAN PHASE WITH LIMITED SUCCESS}

It has been a working assumption of chronobiologists that the harms of shift-worker dysrhythmia would be most effectively mitigated if, instead of altering activity rhythms volitionally or pharmacologically, the phase of the circadian pacemaker and its outputs could be reset to correspond with the desired schedule. In other words, the timing of subjective day and night would be directly shifted, eliminating the dysrhythmia. The phase-shift is thus the most commonly attempted circadian manipulation, and typically involves the timed application of bright light, the principal 


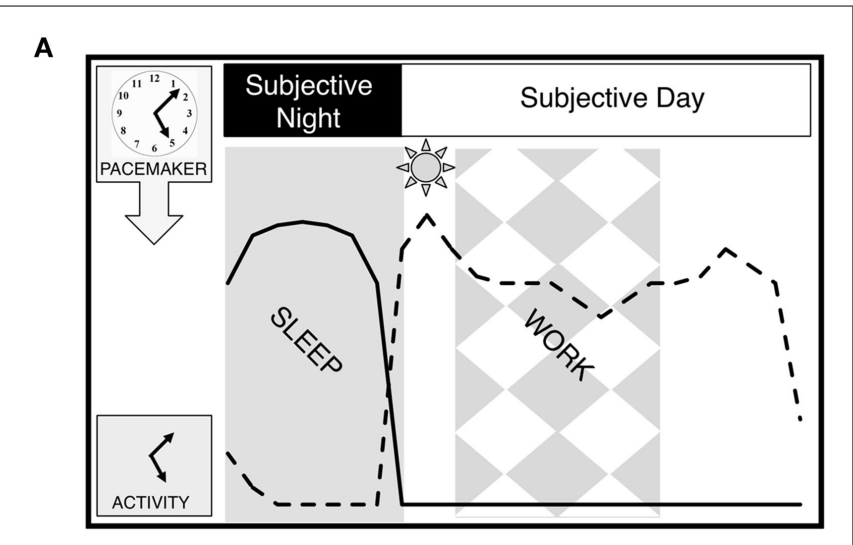

B

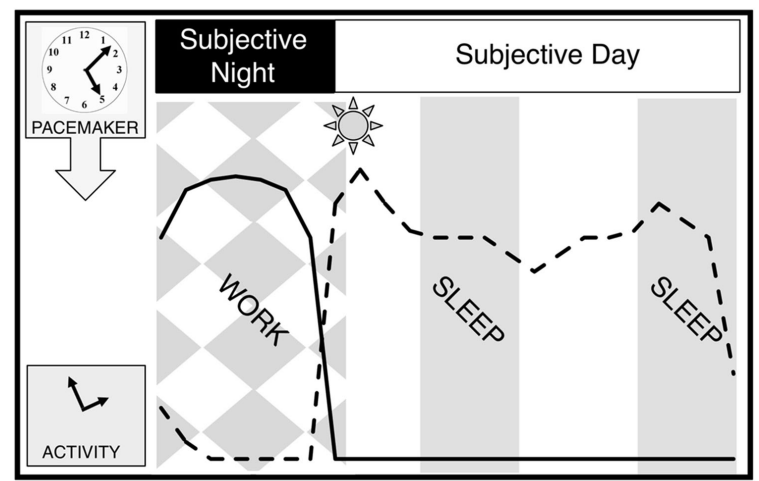

C

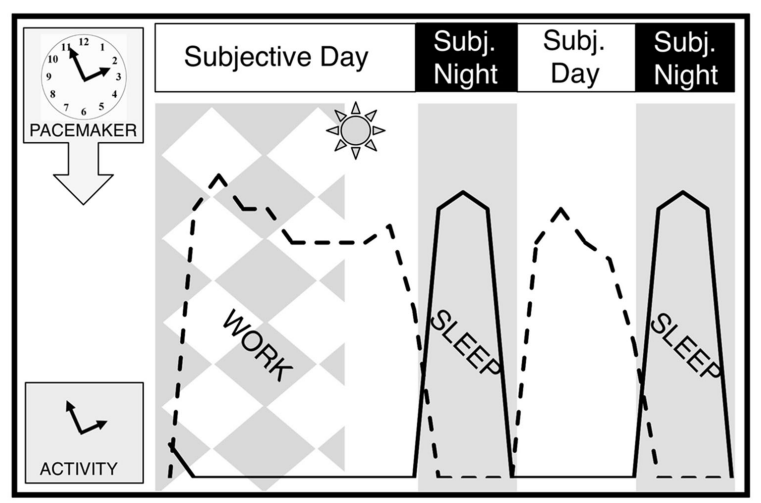

FIGURE 1 | Entrainment and dysrhythmia. (A) Normal entrainment with day work. Sleep coincides with subjective night when melatonin is elevated (solid line) and alertness is low. Work occurs in subjective day when alertness (dashed line) is elevated and melatonin is basal. Each panel begins at midnight of the environmental day. The pacemaker is represented as a clock, while activity, an output of the pacemaker, is represented as the hands of a clock. (B) Dysrhythmia in shift-work. Work is scheduled to coincide with an unaltered subjective night when melatonin is elevated. Sleep is commonly divided into episodes before and after the work shift, but efficiency is low during the subjective day. Behavior is thus out of synchrony with underlying pacemaker. (C) Hypothetical bifurcated entrainment to shift-work. With subjective day and night bifurcated into two components each with presumed changes in alertness, sleep efficiency and melatonin secretion, work could be profitably scheduled during environmental nighttime hours. The worker's night shift now occurs during the first and longer subjective day, with a second subjective day in which to conduct business and interact with family. The light between bouts, which includes morning light, stabilizes the bifurcation (see Pacemaker Bifurcation Potentially Addresses Problems Posed by Shift-Work and Figure 2A for

(Continued)

\section{FIGURE 1 | Continued}

more detail). Sleep occurs in two episodes bracketing a short subjective day in which to interact with family or conduct business. Behavior is always consistent with underlying pacemaker phase. Note that the schedule illustrated here involves a shift of both subjective nights into the former subjective day. Other schedules (e.g., split shifts etc) would not necessarily require this inversion.

environmental cue for resetting all mammalian clocks (Duffy et al., 1996; Danilenko et al., 2000; Crowley et al., 2003; Roenneberg and Merrow, 2007). Under laboratory conditions, humans and nonhuman animals can phase-shift successfully in either direction (earlier or later). In the real world too, humans rapidly crossing several time zones eventually adopt a phase aligned with the new location, though it may take several days. This sluggish rate underscores a significant barrier to effective phase-resetting in the work context: many shift-workers are on rotating shifts, requiring that the pacemaker be shifted both rapidly and repeatedly. Likewise, when a shift-worker is given time off, as on a weekend, the new schedule competes with the old (Smith et al., 2009). In fact, it has been advised for night shift-workers with weekends off to adapt a "compromise phase position," entraining to a phase between the two conflicting schedules, without being well-matched to either (Lee et al., 2006; Smith and Eastman, 2008).

A further complication of phase manipulations is that effective phase-shifting may require complex schedules of light (Revell and Eastman, 2005; Dumont et al., 2009). In humans and other animals, the phase-resetting actions of light are greatest during subjective night: light at the beginning and end of subjective night delays and advances the pacemaker, respectively (Aschoff, 1999). Inappropriately timed light can therefore not only undo an achieved phase-shift, but actually move an individual's clock further from the desired schedule. In particular, bright morning light at the end of a night shift has strong phase-advancing effects that counteract the desired shift (Crowley et al., 2003). Empirically, even in individuals with permanent schedules, for whom a stably altered phase may be reasonably expected, the clock generally remains entrained to a phase typical of day workers. Only one in four shift-workers with a permanent schedule outside of the regular nine to five workday are able to even partially shift circadian phase as measured by melatonin, while only $3 \%$ fully shift (Folkard, 2008). Thus, despite a solid understanding of how the human circadian rhythm responds to light, the lack of practically implemented strategies for shift-workers underscores the need for an alternative target of circadian manipulation that may be more responsive and more tractable than phase.

\section{CIRCADIAN WAVEFORM IS A PROMISING TARGET OF CIRCADIAN MANIPULATION}

The waveform of a circadian rhythm is simply its shape across the $24 \mathrm{~h}$ cycle. Compared to phase (or period), waveform has received scant attention in the circadian literature (e.g., in PubMed, "circadian" and "waveform" yield under $2 \%$ of the hits generated by "circadian" and "phase"). The novel thesis of this "Perspective" paper is that the waveform of the mammalian pacemaker itself is flexible and thus may offer a superior entrainment alternative for shift-workers compared to shifting the phase of the pacemaker or 


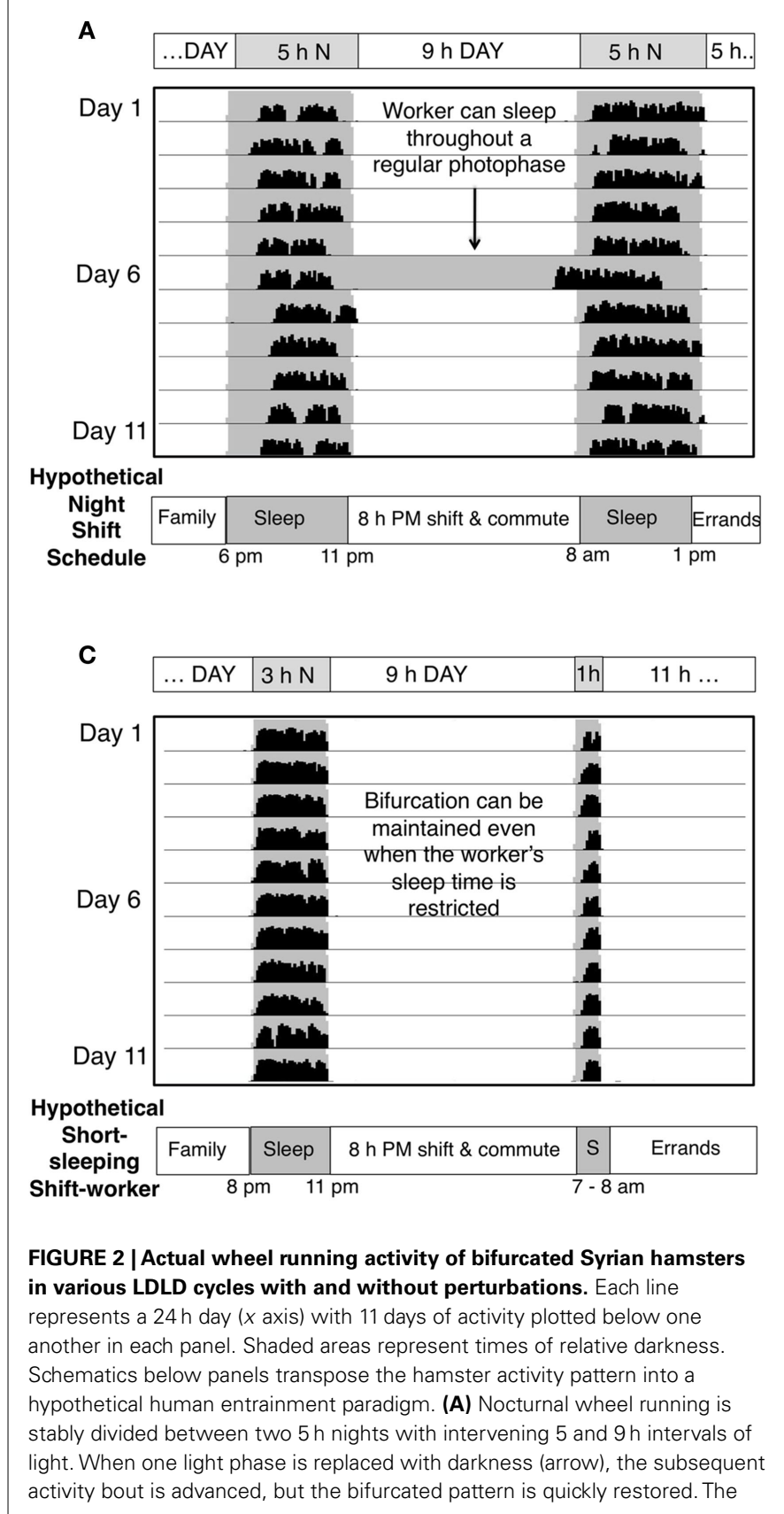

superficially altering the waveform of individual outputs such as sleep/wake.

The impetus for a focus on circadian waveform is a recent series of studies in rodents demonstrating a surprising degree of pacemaker plasticity that can be easily manipulated experimentally (Gorman and Elliott, 2003, 2004). Briefly, when maintained on a permissive $24 \mathrm{~h}$ light:dark:light:dark (LDLD) cycle, nocturnal rodents can be induced to re-organize their circadian timing systems such that they show night-typical levels of activity in each of the two daily dark periods (Figure 2). Likewise, they exhibit daytime patterns of inactivity during each of the two daily light periods. Called rhythm "bifurcation," this entrainment paradigm
B

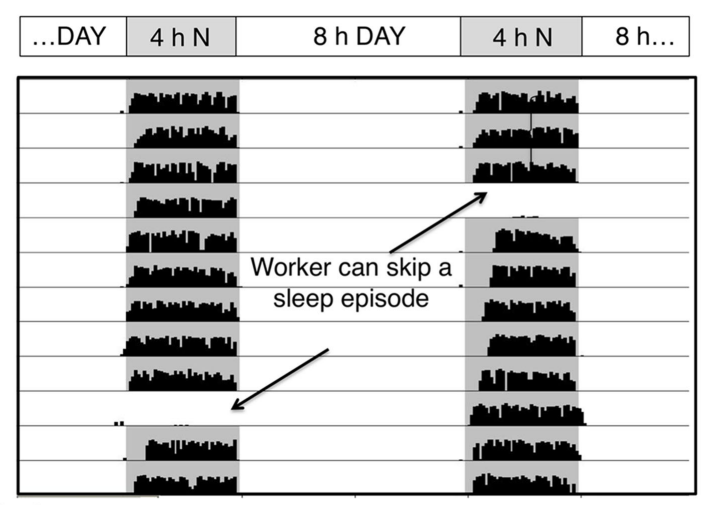

Hypothetical

\begin{tabular}{c|c|c|c|c|c|}
$\begin{array}{c}\text { Watch } \\
\text { Shift }\end{array}$ & $4 \mathrm{~h}$ shift & Sleep & $4 \mathrm{~h}$ shift \& $4 \mathrm{~h}$ free time & Sleep & Family \\
Schedule & \multicolumn{2}{c|}{$6 \mathrm{pm} \quad 10 \mathrm{pm}$} & \multicolumn{2}{c|}{$6 \mathrm{am}$} & $\mathrm{am}$
\end{tabular}

D

\begin{tabular}{|l|c|c|c|c|}
\hline ...DAY & $4 \mathrm{~h} \mathrm{~N}$ & $8 \mathrm{~h}$ DAY & $4 \mathrm{~h} \mathrm{~N}$ & $8 \mathrm{~h} . .$. \\
\hline
\end{tabular}

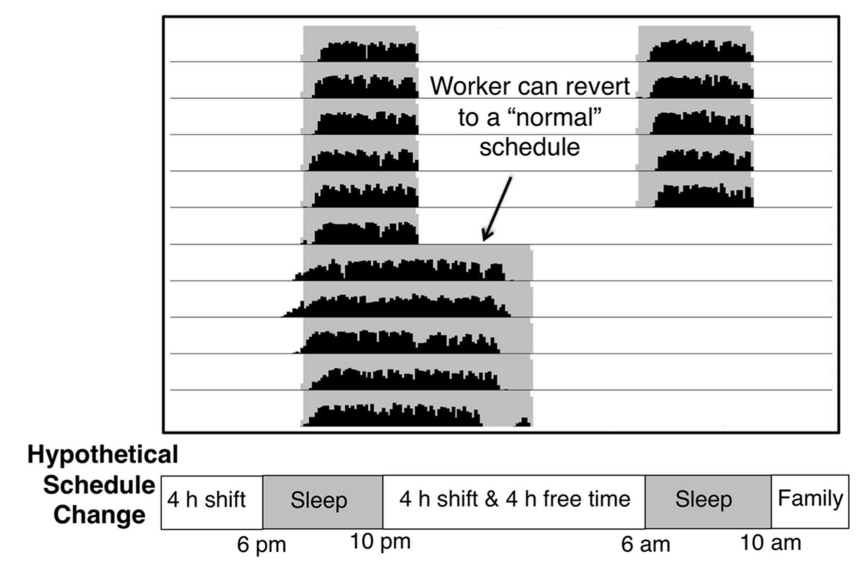

analogous human exposure would be a shift-worker sleeping through the environmental night on a day off or having a long sleep during the day. Note that scotophases do not have to be evenly spaced within $24 \mathrm{~h}$. (B) Removal of a photophase (A) - analogous to a shift-worker sleeping in on a day off-or a scotophase (B) - analogous to a shift-worker staying up all day on a day off-does not compromise the stability of the bifurcated state of entrainment. (C) Scotophases do not have to be of equal length, and bifurcation remains stable even when the bouts are asymmetrical and greatly reduced in length. (D) Bifurcated animals can revert to a normal, diurnal rhythm within one cycle. allows animals to express in each $24 \mathrm{~h}$ cycle two subjective days each lasting $\sim 7-8 \mathrm{~h}$ and beginning $12 \mathrm{~h}$ apart and two subjective nights of $\sim 4-5 \mathrm{~h}$ each, also $12 \mathrm{~h}$ apart. Considerable evidence establishes that this is a bona fide bifurcation of subjective day and night, not a superimposition of an additional activity bout in the middle of subjective day: classic markers of subjective night - melatonin secretion and circadian sensitivity to light pulses - are present during both intervals of activity (Raiewski, under review; Gorman et al., 2001). Thus, in this paradigm, activity rhythms can be temporally reorganized via a change in the waveform of the circadian pacemaker. Mechanistically, bifurcation derives from a temporal dissociation of component oscillators in the core and shell of the 
SCN that appear to correspond to the alternating bouts of activity (Gorman et al., 2001; Watanabe et al., 2007; Yan et al., 2010). This distinguishes it from the splitting paradigm seen in constant light (de la Iglesia et al., 2000).

It remains to be determined whether and how a similar bifurcation of pacemaker waveform might be accomplished in humans, but the implications for shift-workers are nevertheless worth considering. As with any circadian generalization from nocturnal rodents to diurnal humans, this exercise necessitates the transposition of rest/activity relative to subjective day/night. Applying this paradigm to a worker on a stable night shift (Figure 1C), we envision entraining a person to begin one of their two mini-subjective days around midnight coinciding with the beginning of the work shift. After work, a mini-subjective night would allow a $4 \mathrm{~h}$ sleep interval. Waking in the afternoon, the worker would have a second subjective day during daylight hours, and could sleep again during the second mini-subjective night before work. Of course, shift-workers commonly adopt some version of this sleep/wake cycle (Akerstedt and Wright, 2009), as do some who take naps or siestas, but presumably do so without the benefit of a shift in the pacemaker's programming of subjective day and night.

\section{PACEMAKER BIFURCATION POTENTIALLY ADDRESSES PROBLEMS POSED BY SHIFT-WORK}

Bifurcation may offer several significant advantages over an exclusive phase-shifting strategy. First, because it would align the pacemaker with the behaviorally modified output of the sleep/wake cycle (Figure 1C), there is the strong expectation that alertness would be optimized both during the nighttime work shift and the active-time away from work, despite these times falling $12 \mathrm{~h}$ apart at opposite phases of the natural day. Correspondingly, the programming of sleep during intervals of subjective night would plausibly enhance sleep efficiency. The non-pharmacological enhancement of worker alertness and sleep efficiency has obvious benefits related to optimization of productivity and minimization of accidents, and perhaps the physiological and behavioral problems commonly seen in the shift-working population. These suppositions would be subject to rigorous empirical test in bifurcated human subjects.

Further potential benefits of bifurcation relate to scheduling flexibility. As discussed, phase-shifts are generally achieved very slowly in humans, requiring successive timed bright light exposures to generate a controlled shift in entrainment. Bifurcation, in contrast, can be reliably elicited in rodents within a single cycle in nearly every individual without transient entrainment states (Yan et al., 2010). Moreover, the paradigm takes advantage of morning light, which typically impedes entrainment of the shiftworker, but actually reinforces the bifurcated entrainment pattern (Gorman and Steele, 2006). When light between bouts, which includes morning light, is removed, the subsequent activity bout is advanced ("pulled") toward the preceding (Figure 2A). The two bouts will rejoin if the change is made permanent. Thus, the light surrounding the subjective night serves to hold the system in place.

For a bifurcated entrainment protocol to be useful to human shift-workers it must be robust to schedule variation. While days off tend to undo shifts of non-bifurcated rhythms (Smith and Eastman, 2008), bifurcation is immediately re-established after perturbations in the lighting cycle, including replacement of light with darkness (Gorman and Steele, 2006) or darkness with light (Figures 2A,B). If desired, the bifurcated state can, however, be instantly reverted to the unbifurcated state upon exposure to a unimodal LD cycle (Figure 2D). Additionally, the LDLD schedule described above is only one of many that support bifurcated entrainment. The two intervals of darkness in each $24 \mathrm{~h}$ can range anywhere from 1 to $6 \mathrm{~h}$; the two dark periods need not be of equal duration (Figure 2C; unpublished observations), nor occur $12 \mathrm{~h}$ apart (Gorman and Steele, 2006). An asymmetrical bifurcated entrainment schedule provides a good model for a night shift-worker who might commute for an hour before and after an $8 \mathrm{~h}$ work shift to necessitate one $10 \mathrm{~h}$ subjective day. The remaining portion of the $24 \mathrm{~h}$ cycle might be entrained to yield two $4 \mathrm{~h}$ sleep bouts bracketing another $6 \mathrm{~h}$ subjective day for business and family.

\section{THERE IS PRECEDENT FOR PLASTICITY IN PACEMAKER WAVEFORM IN RODENTS AND HUMANS}

If the waveform of mammals is fundamentally plastic, why, then, are there no reports of bifurcated humans in the literature? Specific and surprising environmental conditions, such as dim nocturnal illumination, have been shown to facilitate bifurcation in rodents (Gorman et al., 2003). So we expect that catalytic conditions may also be necessary for bifurcation in humans. Moreover, as in the case of photoperiodism (see below), competing Zeitgebers ("timegivers"), such as bright light at night, may make it difficult for such a reorganization to occur spontaneously. Finally, entrainable pacemaker bifurcation has been described only recently in animal models and to our knowledge has not yet been systematically sought in humans.

Although examinations of bifurcated work schedules might inform the feasibility of human circadian bifurcation, such studies are relatively few and most do not assess melatonin for a definitive determination of subjective night. Unfortunately, they typically focus on the sleep/wake rhythm even though this output is often forced to disassociate and underlies the symptoms of dysrhythmia (Eriksen et al., 2006; Jay et al., 2006; Gander et al., 2008). As discussed, shift-workers commonly split their sleep/wake schedule in an attempt to adjust to the challenges of working odd hours (Akerstedt, 1998a,b). Prophylactic napping is advised as a coping mechanism for shift-work (Akerstedt, 1998a; Sack et al., 2007; Caldwell et al., 2008) and coupled with dark exposure may even promote pacemaker shifting (Weibel et al., 1997). Likewise, polyphasic sleep (including napping and "siestas") is common in many cultures (Webb and Dinges, 1989). These schedules, however, differ from rodent bifurcation in that the waveform of the pacemaker itself is not altered. In the one known study of a split work schedule wherein both sleep and melatonin were measured, the subjects' rest/activity cycle was forced to split while the light schedule remained a natural solar day, and the melatonin waveform remained unimodal (Arendt, 2006). The absence of pacemaker bifurcation under these conditions, while informative, should not be interpreted as indicating that pacemaker bifurcation is impossible.

In these early days of understanding bifurcation requirements, mechanisms, and sequelae, feasibility of rhythm bifurcation in 
humans is more reasonably evaluated with respect to fundamentals of circadian organization. One context in which circadian waveform has been studied is the entrainment to seasonally varying daylengths (i.e., photoperiodism). Pacemaker waveform has been commonly conceptualized as a simple alternation (e.g., square-wave) between biological night and day, and both humans and non-human animals share a capacity for waveform adjustment to seasonal changes in relative length of night. In rodents, the waveform of a given photoperiod is encoded in the SCN itself (Schwartz et al., 2001; Sumová et al., 2004; Meijer et al., 2010) with parallel patterns in such downstream outputs as melatonin and behavior (Illnerova et al., 1984; Elliott and Tamarkin, 1994; Figure 2C).

Humans retain this fundamental photoperiodic capacity: the duration of human nocturnal melatonin secretion can expand in both natural and artificial daylength manipulations (Beck-Friis et al., 1984; Kauppila et al., 1987; Makkison and Arendt, 1991; Wehr, 1991, 1998; Stokkan and Reiter, 1994; Vondrasova et al., 1997). The waveform of human melatonin can be considered an index of the waveform of the underlying pacemaker (Lewy, 1999). Moreover, that the extension of melatonin persists in constant conditions (Wehr, 1991; Wehr et al., 1993) indicates a change in the underlying pacemaker and provides evidence that humans retain the machinery required to alter circadian waveform in response to environment. Human photoperiodism therefore represents a naturally occurring and evolutionarily conserved manipulation of pacemaker waveform. Parenthetically, it is not commonly seen in urban populations, likely because most individuals substitute artificial for bright, natural sunlight (Savides et al., 1986; Hébert et al., 1998), and artificially extend their days with indoor lighting (Wehr et al., 1995a).

Although direct measures of SCN function are not possible in humans, all indications are that circadian system organization is conserved: cross-species commonalities exist in fundamental properties of circadian systems, such as molecular basis of cellular rhythmicity, SCN cell types and neuroanatomy, circadian control of organ systems, and phase-resetting actions of light (Hofman et al., 1996; Duffy and Wright, 2005). Further indications of multi-oscillator circadian function in humans include bimodality in rhythms of alertness, performance, and errors across $24 \mathrm{~h}$; (Campbell and Zulley, 1985; Mitler et al., 1988; Bes et al., 2009) bimodal sleep and melatonin secretion (Arendt, 1985; Wehr et al., 1995b), and dissociable rhythmic components seen under internal desynchrony protocols (Aschoff and Wever, 1976; Folkard and Wever, 1983). Given these commonalities the presumption should be that the human pacemaker, like those of other mammals, will be dissociable under permissive conditions.

\section{REFERENCES}

Akerstedt, T. (1998a). Is there an optimal sleep-wake pattern in shift work? Scand. J. Work Environ. Health 24(Suppl. 3), 18-27.

Akerstedt, T. (1998b). Shift work and disturbed sleep/wakefulness. Sleep Med. Rev. 2, 117-128.

Akerstedt, T., and Wright, K. P. Jr. (2009). Sleep loss and fatigue in shift work and shift work disorder. Sleep Med. Clin. 4, 257-271.

Arendt, J. (1985). Mammalian pineal rhythms. Pineal Res. Rev. 3, 161-374. Arendt, J. (2006). Melatonin and human rhythms. Chronobiol. Int. 23, 21-37.

Aschoff, J. (1999). Masking and parametric effects of high-frequency light-dark cycles. Jpn. J. Physiol. 49, 11-18.

\section{THE HUMAN SLEEP/WAKE CYCLE IS FLEXIBLE AND MAY NOT HINDER BIFURCATION}

Although application of a bifurcated entrainment pattern to humans has been motivated by attractive features of having two alertness intervals per $24 \mathrm{~h}$ cycle, a necessary consequence is that the human nocturnal sleep pattern would likewise be bifurcated. This may cause some concern among sleep experts as, in general, consolidated sleep is considered preferable to fragmented sleep (Bonnet, 1986; Stepanski, 2002) and daytime sleep is poorer in quality than nighttime sleep (Dijk et al., 1999; Akerstedt and Wright, 2009). However, such principles consider daytime sleep in the context of a normal, diurnal, and unimodal waveform. The incidence of two subjective nights in which to sleep might circumvent these issues, which are likely a result of sleep occurring during subjective day. In fact, assessments of vigilance in monophasic versus polyphasic sleep schedules indicate that performance is comparable given equivalent time in bed (Nicholson et al., 1985; Mollicone et al., 2007; Mollicone, 2008). Moreover, polyphasic sleep can be more effective than monophasic sleep in combating the buildup of fatigue in sleep-deprived subjects (Hartley, 1974). Therefore, performance may improve under bifurcation, though any effects on sleep and other physiological outcomes are yet unknown.

\section{SUMMARY}

Photoperiodic responses in human melatonin that persist in constant conditions suggest that the human pacemaker itself is responsive to experimental manipulations of waveform. The bimodality of output rhythms suggests that there are potentially dissociable components in the human pacemaker and thus results from animal studies of rhythm waveform manipulation may generalize to humans. Taken together, these findings allude to the potential for a more complete adjustment to challenging schedules given appropriate Zeitgebers. Experimental manipulation of the waveform of the human pacemaker, a largely neglected circadian parameter, may therefore have the potential to vastly improve upon current methodologies for treating dysrhythmia, thereby reducing the health, safety, and performance costs of shift-work. Future research should focus on characterizing the optimal and minimal protocols to induce such plasticity in animal models, while simultaneous inquiries into the feasibility of introducing such protocols into human research are explored.

\section{ACKNOWLEDGMENTS}

The authors are grateful to Jeffrey Elliott and Gena Glickman for comments on earlier drafts of the manuscript. Supported by NICHD grant 36460 (Michael R. Gorman).

Aschoff, J., and Wever, R. (1976). Human circadian rhythms: a multioscillatory system. Fed. Proc. 35, 236-268.

Beck-Friis, J., Von Rosen, D., Kjellman, B. F., Ljunggren, J. G., and Wetterberg, L. (1984). Melatonin in relation to body measures, sex age, season and the use of drugs in patients with major affective disorders and healthy subjects. Psychoneuroendocrino. 9, 261-277.

Bes, F., Jobert, M., and Schulz, H. (2009). Modeling napping, post-lunch dip, and other variations in human sleep propensity. Sleep 32, 392-398.

Bonnet, M. (1986). Performance and sleepiness as a function of frequency and placement of sleep disruption. Psychophysiology 23, 263-334. 
Caldwell, J., Caldwell, J., and Schmidt, R. (2008). Alertness management strategies for operational contexts. Sleep Med. Rev. 12, 257-330.

Campbell, S. S., and Zulley, J. (1985). "Ultradian components of human sleep/wake patterns during disentrainment," in Ultradian Rhythms in Physiology and Behavior, eds P. Lavie and H. Schulz (Berlin: SpringerVerlag), 234.

Costa, G. (1996). The impact of shift and night work on health. Appl. Ergon. 27, 9-25.

Costa, G., Haus, E., and Stevens, R. (2010). Shift work and cancer - considerations on rationale, mechanisms, and epidemiology. Scand. J. Work Environ. Health 36, 163-179.

Crowley, S. J., Lee, C., Tseng, C. Y., Fogg, L. F., and Eastman, C. I. (2003). Combinations of bright light, scheduled dark, sunglasses, and melatonin to facilitate circadian entrainment to night shift work. J. Biol. Rhythms 18, 513-523.

Danilenko, K. V., Wirz-Justice, A., Kräuchi, K., Cajochen, C., Weber, J. M., Fairhurst, S., and Terman, M. (2000). Phase advance after one or three simulated dawns in humans. Chronobiol. Int. 17, 659-668.

Davidson, A. J., Sellix, M. T., Daniel, J., Yamazaki, S., Menaker, M., and Block, G. D. (2006). Chronic jetlag increases mortality in aged mice. Curr. Biol. 16, R914-R916.

de la Iglesia, H. O., Meyer, J. H., Carpino, A. Jr., and Schwartz, W. J. (2000). Antiphase oscillation of the left and right suprachiasmatic nuclei. Science 290, 799-801.

Dijk, D., Duffy, J., Riel, E., Shanahan, T., and Czeisler, C. (1999). Ageing and the circadian and homeostatic regulation of human sleep during forced desynchrony of rest, melatonin and temperature rhythms. J. Physiol. (Lond.) 516(Pt 2), 611-638.

Duffy, J. F., Kronauer, R. E., and Czeisler, C. A. (1996). Phase-shifting human circadian rhythms: influence of sleep timing, social contact and light exposure. J. Physiol. (Lond.) 495(Pt 1), 289-297.

Duffy, J. F., and Wright, K. P. Jr. (2005). Entrainment of the human circadian system by light. J. Biol. Rhythms 20, 326-338.

Dumont, M., Blais, H., Roy, J., and Paquet, J. (2009). Controlled patterns of daytime light exposure improve circadian adjustment in simulated night work. J. Biol. Rhythms 24, 427-437.
Dunlap, J. C., Loros, J. J., and Decoursey, P. J. (2004). Chronobiology: Biological Timekeeping. Sunderland, MA: Sinauer Associates.

Elliott, J. A., and Tamarkin, L. (1994). Complex circadian regulation of pineal melatonin and wheelrunning in Syrian hamsters. $J$. Comp. Physiol. A 174, 469-484.

Eriksen, C., Gillberg, M., and Vestergren, P. (2006). Sleepiness and sleep in a simulated "six hours on/six hours off" sea watch system. Chronobiol. Int. 23, 1193-1395.

Folkard, S. (2008). Do permanent night workers show circadian adjustment? A review based on the endogenous melatonin rhythm. Chronobiol. Int. 25, 215-224.

Folkard, S., and Tucker, P. (2003). Shift work, safety and productivity. Occup. Med. (Lond.) 53, 95-196.

Folkard, S., and Wever, R. (1983). Multi-oscillatory control of circadian rhythms in human performance. Nature 305, 223-226.

Gander, P., Van Den Berg, M., and Signal, L. (2008). Sleep and sleepiness of fishermen on rotating schedules. Chronobiol. Int. 25, 389-398.

Golombek, D., and Rosenstein, R (2010). Physiology of circadian entrainment. Physiol. Rev. 90, 1063-1165.

Gorman, M. R., and Elliott, J. A. (2003). Entrainment of 2 subjective nights by daily light:dark:light:dark cycles in 3 rodent species. J. Biol. Rhythms $18,502-512$.

Gorman, M. R., and Elliott, J. A. (2004). Dim nocturnal illumination alters coupling of circadian pacemakers in Siberian hamsters, Phodopus sungorus. J. Comp. Physiol. A Neuroethol. Sens. Neural Behav. Physiol. 190, 631-639.

Gorman, M. R., Elliott, J. A., and Evans, J. A. (2003). Plasticity of hamster circadian entrainment patterns depends on light intensity. Chronobiol. Int. 20, 233-248.

Gorman, M. R., and Steele, N. A. (2006). Phase angle difference alters coupling relations of functionally distinct circadian oscillators revealed by rhythm splitting. J. Biol. Rhythms 21, 195-205.

Gorman, M. R., Yellon, S. M., and Lee, T. M. (2001). Temporal reorganization of the suprachiasmatic nuclei in hamsters with split circadian rhythms. J. Biol. Rhythms 16, 552-563.

Hansen, J. (2001). Light at night, shiftwork, and breast cancer risk. J. Natl. Cancer Inst. 93, 1513-1518.

Hartley, L. R. (1974). A comparison of continuous and distributed reduced sleep schedules. Q. J. Exp. Psychol.26, 8-14.

Hébert, M., Dumont, M., and Paquet, J. (1998). Seasonal and diurnal patterns of human illumination under natural conditions. Chronobiol. Int $15,59-70$.

Hofman, M. A., Zhou, J. N., and Swaab, D. F. (1996). No evidence for a diurnal vasoactive intestinal polypeptide (VIP) rhythm in the human suprachiasmatic nucleus. Brain Res. 722, 78-82.

Illnerova, H., Hoffmann, K., and Vanecek, J. (1984). Adjustment of pineal melatonin and $\mathrm{N}$ acetyltransferase rhythms to change from long to short photoperiod in the Djungarian hamster Phodopus sungorus. Neuroendocrinology 38 226-231.

Jay, S. M., Dawson, D., and Lamond, N. (2006). Train drivers' sleep quality and quantity during extended relay operations. Chronobiol. Int. 23 , 1241-1252.

Kauppila, A., Kivelä, A., Pakarinen, A. and Vakkuri, O. (1987). Inverse seasonal relationship between melatonin and ovarian activity in humans in a region with a strong seasonal contrast in luminosity. J. Clin Endocrinol. Metab. 65, 823-828.

Kerin, A., and Aguirre, A. (2005) Improving health, safety, and profits in extended hours operations (shiftwork). Ind. Health 43, 201-209.

Lee, C., Smith, M. R., and Eastman, C. I. (2006). A compromise phase position for permanent night shift workers: circadian phase after two night shifts with scheduled sleep and light/dark exposure. Chronobiol. Int 23, 859-875.

Lewy, A. J. (1999). Melatonin as a marker and phase-resetter of circadian rhythms in humans. Adv. Exp. Med. Biol. 460, 425-434.

Makkison, I., and Arendt, J. (1991). Melatonin secretion in humans on two different Antarctic bases (68 and 75 S). J. Interdiscipl. Cycle Res. 22, 149-150.

Meijer, J. H., Michel, S., Vanderleest, H. T., and Rohling, J. H. T. (2010) Daily and seasonal adaptation of the circadian clock requires plasticity of the SCN neuronal network. Eur. J. Neurosci. 32, 2143-2151.

McMenamin, T. (2007). Time to work: recent trends in shift work and flexible schedules, A. Monthly Lab. Rev. 130, 3-15.

Mitler, M., Carskadon, M., Czeisler, C. Dement, W., Dinges, D., and Graeber, R. (1988). Catastrophes, sleep and public policy: consensus report. Sleep 11, 100-109.
Mollicone, D. (2008). Sleep and Neurobehavioral Performance during a 14-day Laboratory Study of Split Sleep/Wake Schedules for Space Operations. Dissertation, 1-186.

Mollicone, D. J., Van Dongen, H. A., and Dinges, D. F. (2007). Optimizing sleep/wake schedules in space: sleep during chronic nocturnal sleep restriction with and without diurnal naps. Acta Astronaut. 60, 354-361.

Nicholson, A. N., Pascoe, P. A., Roehrs, T., Roth, T., Spencer, M. B., Stone, B. M., and Zorick, F. (1985). Sustained performance with short evening and morning sleeps. Aviat. Space Environ. Med. 56, 105-114.

Revell, V., and Eastman, C. (2005). How to trick mother nature into letting you fly around or stay up all night. $J$. Biol. Rhythms 20, 353-418.

Roenneberg, T., and Merrow, M. (2007). Entrainment of the human circadian clock. Cold Spring Harb. Symp. Quant. Biol. 72, 293-299.

Rosekind, M. (2005). Underestimating the societal costs of impaired alertness: safety, health and productivity risks. Sleep Med. 6(Suppl. 1), S21-S25.

Sack, R., Auckley, D., Auger, R. Carskadon, M., Wright, K., Vitiello, M., Zhdanova, I., and American Academy of Sleep Medicine. (2007). Circadian rhythm sleep disorders: part I, basic principles, shift work and jet lag disorders. An American Academy of Sleep Medicine review. Sleep 30, 1460-1543.

Savides, T. J., Messin, S., Senger, C., and Kripke, D. F. (1986). Natural light exposure of young adults. Physiol. Behav. 38, 571-574.

Schwartz, W. J., De La Iglesia, H. O., Zlomanczuk, P., and Illnerová, $\mathrm{H}$. (2001). Encoding le quattro stagioni within the mammalian brain: photoperiodic orchestration through the suprachiasmatic nucleus. J. Biol. Rhythms 16, 302-311.

Smith, M. R., and Eastman, C. I. (2008). Night shift performance is improved by a compromise circadian phase position: study 3 . Circadian phase after 7 night shifts with an intervening weekend off. Sleep 31 , 1639-1645.

Smith, M. R., Fogg, L. F., and Eastman, C. I. (2009). Practical interventions to promote circadian adaptation to permanent night shift work: study 4. J. Biol. Rhythms 24, 161-172.

Stepanski, E. (2002). The effect of sleep fragmentation on daytime function. Sleep 25, 268-344. 
Stokkan, K. A., and Reiter, R. J. (1994). Melatonin rhythms in arctic urban residents. J. Pineal Res. 16, 33-36.

Sumová, A., Bendová, Z., Sládek, M., Kováciková, Z., and Illnerová, $\mathrm{H}$. (2004). Seasonal molecular timekeeping within the rat circadian clock. Physiol. Res. 53(Suppl 1), S167-S176.

Vondrasova, D., Hajek, I., and Illnerova, H. (1997). Exposure to long summer days affects the human melatonin and cortisol rhythms. Brain Res. 759, 166-170.

Watanabe, T., Naito, E., Nakao, N., Tei, H., Yoshimura, T., and Ebihara, S. (2007). Bimodal clock gene expression in mouse suprachiasmatic nucleus and peripheral tissues under a 7-hour light and 5-hour dark schedule. J. Biol. Rhythms 22, 58-68.

Webb, W. B., and Dinges, D. F. (1989). "Cultural perspectives on napping and the siesta," in Sleep and Alertness, eds D. F. Dinges and R. J. Broughton (New York, NY: Raven Press), 247-312.

Wehr, T. A. (1991). The durations of human melatonin secretion and sleep respond to changes in daylength (photoperiod). J. Clin. Endocrinol. Metab. 73, 1276-1280.

Wehr, T. A. (1998). Effect of seasonal changes in daylength on human neuroendocrine function. Horm. Res. 49, 118-124.

Wehr, T. A., Giesen, H., Moul, D., Turner, E., and Schwartz, P. (1995a). Suppression of men's responses to seasonal changes in day length by modern artificial lighting. Am. J. Physiol. 269, 173-178.

Wehr, T. A., Schwartz, P. J., Turner, E. H., Feldman-Naim, S., Drake, C. L., and Rosenthal, N. E. (1995b). Bimodal patterns of human melatonin secretion consistent with a two-oscillator model of regulation. Neurosci. Lett. 194, 105-108.

Wehr, T. A., Moul, D. E., Barbato, G., Giesen, H. A., Seidel, J. A., Barker, C., and Bender, C. (1993). Conservation of photoperiod-responsive mechanisms in humans. Am. J. Physiol. 265, R846-R857.

Weibel, L., Spiegel, C., Gronfier, C., Follenius, M., and Brandenberger, G. (1997). Twenty-four-hour melatonin and core body temperature rhythms: their adaptation in night workers. Am. J. Physiol. 272, R948-R954.

Yan, L., Silver, R., and Gorman, M. (2010). Reorganization of suprachiasmatic nucleus networks under 24-h LDLD conditions. J. Biol. Rhythms 25, 19-27.

Conflict of Interest Statement: The authors declare that the research was conducted in the absence of any commercial or financial relationships that could be construed as a potential conflict of interest.

Received: 22 February 2012; paper pending published: 13 March 2012; accepted: 13 April 2012; published online: 01 May 2012.

Citation: Harrison EM and Gorman $M R$ (2012) Changing the waveform of circadian rhythms: considerations for shift-work. Front. Neur. 3:72. doi: 10.3389/fneur.2012.00072

This article was submitted to Frontiers in Sleep and Chronobiology, a specialty of Frontiers in Neurology.

Copyright (c) 2012 Harrison and Gorman. This is an open-access article distributed under the terms of the Creative Commons Attribution Non Commercial License, which permits non-commercial use, distribution, and reproduction in other forums, provided the original authors and source are credited. 